\title{
The Study of Multi-agent-based Logistics System of Special Items
}

\author{
Ma Ying, Zhu Jianyong \\ National Computer System Engineering Research Institute Of China
}

\begin{abstract}
With the rapid development of information technology, information technology tools have been integrated into the enterprise logistics' management, and achieved good social and economic benefits. But mostly the existing logistics system is for ordinary goods logistics, no one system is designed for special items logistics - bacterial pathogens (viruses) or samples, and this cannot meet the requirements of special items' logistics management. This paper analyzes the importance of the special items' logistics system, combined with Agent technology, proposes a logistics system of special items based on Multi-Agent technology, and describes its architecture and model, to effectively solve the special items logistics and transport issues under the distributed environment.
\end{abstract}

Keywords-Agent, Multi-Agent, Special items logistics

\section{INTRODUCTION}

In order to create a healthy environment, maintaining social stability, more and more public health management and service departments, hospitals, schools, pharmaceutical manufacturers and other units involve in the disease control and research work. The special items - bacterial pathogens (viruses) or samples used for disease control and research with special physical, chemical and infectious characteristics, which belongs to dangerous biological goods, the disclosure will cause harm to human life, the consequences could be disastrous. Therefore establishing a scientific and rational logistics system of special goods can effectively promote the work of disease control and research development.

Like general goods, special items logistics have the same problems,such as protecting the supply, rational using of resources. In addition, different types of laboratories involving different kind special items, and geographically dispersed.Thus it requires distributed management, and the ability of network management. The Agent has the autonomy, responsiveness, social, activity and other characteristics, suitable for the changing system, in where the members are natural distribution, autonomy, and demand flexibility interaction. Obviously the logistics management system of special items is such systems, suitable to description by Agent model.

Distributed Multi-Agent system emphasizes independent decision-making and cooperative interaction between the Agents, which can meet the requirement of special items logistics. But, now the Agent technology used for the logistics management of special items is very little. Based on this idea, this paper proposes a system model of the special items logistics based on Multi-Agent.

\section{AGENT AND Multi-AGENT InTRODUCTION}

\section{A. Agent}

Agent technology dates back to the early artificial intelligence research, in 1970s MIT researchers have developed a series of studies on distributed artificial intelligence [1]. In recent years, Agent system technology has become one of the fastest-growing issues in the field of distributed artificial intelligence and computer science, and introduced to the field of logistics. The biggest feature of Agent technology is that Agent has certain intelligence and a strong ability to interact and conduct free from outside interference.

1) the weak concept of Agent

Michael Wooldridge's definition [2]: computer systems based on hardware or software and with autonomy, social ability, reactivity and initiative nature.

2) the strengthen concept of Agent

Some researchers, particularly in the field of artificial intelligence researchers, hold that the Agent have a more enhanced and more specific meaning. They think: addition to the above attributes, agent are also used often to describe the concept of human conceptualization or implementation of computer systems [3].

3) the basic structure of Agent

Agent is composed of context-aware module, implementation module, communication module, information processing module, decision and control modules, knowledge base and task list, whose structure as shown below [4]:

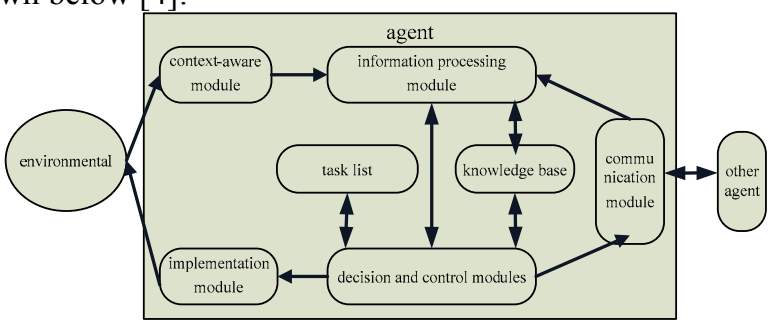

Figure 1. the basic structure of Agent

\section{B. Multi-Agent System}

As a single Agent capability is limited, so commonly used in practical applications is the Multi-Agent system. Multi-Agent system can interact and collaborate with environment and other agents, which is important to solve distributed problem of logistics system. 


\section{1) Multi-Agent System}

MAS (Multi-Agent System) composed of several Agents, by cooperation between Agent members, mutual service and communication to complete a task. Agent members are autonomous and independent.

\section{2) Multi-Agent System Architecture}

MAS can be either entirely composed of relatively small individual agent's federation (the paper referred to: primary MAS), but also can organized by many primary MAS(referred to as the nested MAS).

Primary MAS can be composed of many single agents by centralized or distributed form. Centralized primary MAS has at least one management services, as show in Figure2.

The relationship between the members of distributed primary MAS is equal, and they provide services mutually, as show in Figure3.

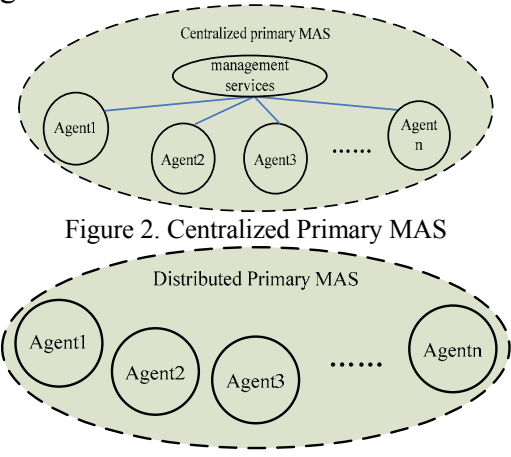

Figure 3. Distributed Primary MAS

Nested MAS is composed of by a number of the primary MAS. So its architecture can be divided into three categories:

(1)Fully centralized MAS: which composed by a number of primary MAS with centralized structure, and all the primary MAS itself is centralized structure, as show in Figure4.

(2)Fully distributed MAS: it consists of several primary MAS by distributed structure, and primary MAS itself is distributed structure, as show in Figure5.

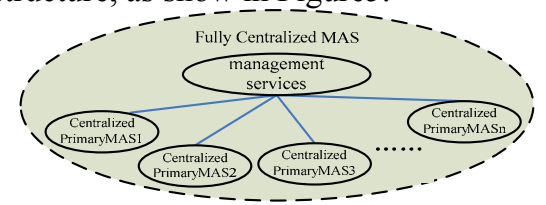

Figure 4. Fully Centralized MAS

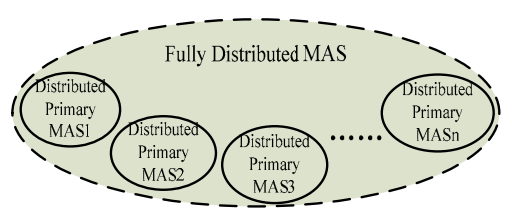

Figure 5. Fully Distributed MAS

(3)Hybrid MAS: which is generally mixed by centralized and distributed primary MAS, as shown below:

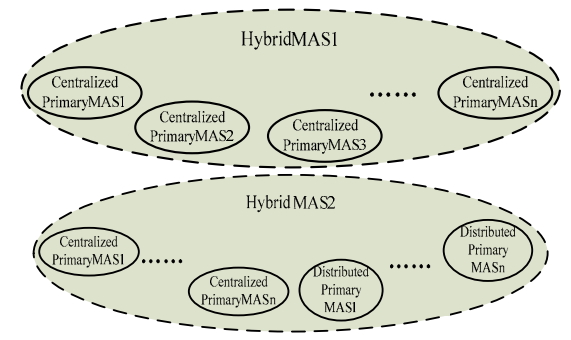

Figure 6. Hybrid MAS

\section{Multi-Agent- BASEd Logistics System Model}

\section{OF SPECIAL ITEMS}

\section{A. Requirements Analysis}

The main work of special items logistics is providing transportation services for the units engaged in the disease prevention and control. The major task is planning, organizing and controlling works during the process of ordering, reserving, transporting and using special items, and the core work is the transportation and preservation management. Its transport process, compared to the ordinary materials logistics, not only demands to solve the rational use of resources, reduce the transport cycle, improve efficiency, and reduce cost, but also needs to pay more attention to its privacy, security, timeliness requirements. The main features of special items' logistics and transportation:

(1) Preservation center, wide regional distribution. The units which engage in the study of disease prevention and control are located in different place, also the study object of each unit are not the same.

(2) Large number and more varieties of bacteria (viruses) species. Bacteria (viruses) refers to culture, human-borne fungi, actinomycetes, bacteria, and ather viruses with conservation value, through the preserved institution identification, classification and give fixed number of micro-organisms. Samples of pathogenic microorganisms (hereinafter referred to as samples) is contain human and animal body fluids, organizations, waste and other substances with conservation value, as well as food and environmental samples. [5]

(3) Security, timeliness demanding. Special items with a high degree of risk, any problems during transport are likely to bring a great loss to the people's lives and property. Therefore, transport process of special items requires very high on safety management, and the timeliness tracking of the logistics information.

So the logistics system of special items should have multiple sources of information, informative, wide range of large-scale, dynamic, complex structures and other features. The Agent has the autonomy, reactivity and other characteristics, suitable for the changing system. Obviously, the logistics system of special items is suitable for Agent model. With the initiative and reactivity feature, the Agent 
is easy to response to the environmental change timely, and improve the management. Based on this background, and according to the needs of business management for special items transport, the paper will establish a logistics system model of special items based on Multi-Agent technology.

\section{B. Multi-Agent-Based Logistics System Model Of Special}

\section{Items}

Because of the distributed characteristics, the logistics system of special goods is very suitable for the application of Multi-Agent technology. We can think the internal mechanism of special items logistics as a system composed of mutual cooperation Agents. Each Agent complete one or more functions of logistics, and each Agent coordinate with others. Elements in the logistics system of special items such as hospitals, health research units, preservation organizations, orders, resources, vehicles, routes, and warehousing can be abstracted as an independent Agent, each Agent has its own intention and behavior, can interacts and coordinates with each other.

There are two construction types of this logistics system model: first, the fully distributed MAS, the other is the hybrid MAS. Two models have advantages and disadvantages. Fully distributed MAS has great flexibility, but the ability to solve global problems slightly less, more demanding on the Agent themselves. Therefore, this paper adopts hybrid MAS structure. The model consists of three layers which are composed of many Agents, as shown below:

Control layer

Figure 7. Multi-Agent-based logistics system model structure of special items

The layers' functions and objectives of this model are as follows:

Control layer: made of the decision-making Agent, has the highest decision-making powers, can assess the transport status of the entire system.

Coordination layer: a middle layer, constituted by the coordination Agents, is responsible for the units' transport within the region, while need to timely transport the situation information in their respective regions to control layer, as a reference for the entire system management

Functional layers: is a bottom layer, composed by the resources Agent, preserved Agent, scheduling and monitoring Agent, in addition, including the main bearer to achieve the transport task, such as vehicles Agent. By the resources using, storage conditions, the situation occurred in the scheduling and real-time monitoring information, they can determine the appropriate transport strategy, also transport the monitoring results and relevant information to coordination layer, as a reference for regional coordination.

\section{Agent Features Of The System Model}

(1) Resource management Agent: manages various resources information, such as adding resources, deleting resources, registering used resources and management, which involves all kinds of information resource of special items' logistics system: includes units, vehicles, drivers, RFID labels. It accepts applications from the resources Agent, rational distribute and use the resources, and returns the resource allocation information.

(2) Preservation management Agent: is primarily responsible for the inventory, access, and state management, and manage the job, files, and users of preservation organizations. The system is divided into preserved management, strain flow management, document managementand resource management.

(3) Scheduling Agent: before special items in the transport, according to the order and road information, make reasonable transportation routes of vehicles and transport plans. Another, dispatch personnel and vehicles can communicate scheduling information at any time, in time supply the information in-transit to vehicles to reduce vehicle no-load rate, so real close to scheduling, real-time scheduling, accurate scheduling, improve work efficiency.

(5) Transport Agent: It is mainly responsible for the management of special goods transportation, the main business are: manage transportation approval, transport packaging, transport and receive.

(4) Monitoring Agent: The Agent is based on GIS, monitors and manages the special items in transit, which receives the vehicle location, cargo status and other information through GPRS, to achieve real-time tracking the location of special items, special items state security monitoring, vehicle trajectory query.

\section{Multi-Agent Interaction And Communication}

\section{1) Multi-Agent Interaction}

For completing the business objectives, Agent in the logistics systems of special items must be mutual cooperation. Resource Agent, preserved Agent, scheduling Agent, transport Agent, monitoring Agent and other elements, not only have its own responsibilities and actions, but also interact with each orher.

When the unit needs to transport, it presents transportation request to the transport Agent, informed requested type, quantity, time and other information. Based on the information which feedback, decision-making Agent decide whether agree this transport request, if agree, it need to notice: preservation Agent for the storage; scheduling Agent for the vehicle, the driver allocation and transportation route choice; transport Agent for transport packaging, transport and receive preparation, and will also 
inform the transportation to applicant. During the transportation process, the monitoring Agent will real-time monitor the location of vehicles and the status of special items, then informs monitoring information to other Agent to prepare to take appropriate action.

2) Agent communication between

In the Multi-Agent-based logistics system of special items, Agents communicate by communication language (ACL) [6], the most commonly things used by communication language are knowledge query and manipulation language (KQML). KQML language model consists of three parts [7]: the content layer, message layer and communication layer, KQML syntax is relatively simple, by a semantic action, a description of the content of expressions and a set of message parameters. This language readable, simple, easy to build, and scalable.it can define new behavior of the original language for different MultiAgent Systems, as long as the specifications of these primitives can be found KQML, KQML as it is chosen here communication mode between the agent.

\section{CONCLUSION}

This paper analyzes the characteristics of special goods logistics- risk, distributed, etc., and introduces the agent technology and multi-agent system. On this basis, this paper proposes the Multi-Agent-based logistics system model of special items. The paper describes demand, the structure of the system model and the functions of modules in detail. In short, through studying the process of setting up MultiAgent-based logistics system of special items, and analyzing this logistics system model, we can see that the studying and developing the model of Multi-Agent-based logistics system of special items have an important application prospect and practical value.

\section{REFERENCES}

[1] Lu Ruling, Knowledge of science and computing science [M]. Beijing: Tsinghua University Press, 2003, 101-102

[2] Michael W, Nicholas R J. Intelligent agents: theory and practice. Knowledge Engineering Review, Vol: 10, Iss: 2, 1995:115-152

[3] Dr.J0hnHunt.BlackboardAichitectures.JayDeeTechnologyLtd.2002

[4] LiYu, Multi-Agent System Architecture and the Study of Agent interaction [D]. Xidian University, 2003 (5)

[5] Human transmission of pathogenic microorganisms' bacteria (viruses) preserved body management. Ministry of Health

[6] Iglesias C A. Analysis and design of multi-Agent systems using MAS-Common KADS.1997:25-36

[7] He Yanxiang, Chen Cuimeng. Agent and Multi-Agent Systems Design and Application. Wuhan: Wuhan University Press .2003:203219 